\section{Estudio de prevalencia de síndrome de apneas obstructivas del sueño en la población adulta chilena. Subestudio de la Encuesta Nacional de Salud, 2016/17}

\author{
FERNANDO SALDÍAS PEÑAFIEL ${ }^{1}$, PABLO BROCKMANN VELOSO ${ }^{2}$, \\ JULIA SANTÍN MARTÍNEZ ${ }^{3}$, EDUARDO FUENTES-LÓPEZ ${ }^{4}$, \\ ISABEL LEIVA RODRÍGUEZ ${ }^{1}$, GONZALO VALDIVIA CABRERA ${ }^{5}$
}

\section{Prevalence of obstructive sleep apnea syndrome in Chilean adults. A sub-study of the national health survey, 2016/17}

Background: Obstructive sleep apnea syndrome (OSAS) affects approximately 10\%-20\% of adults and is associated with obesity, hypertension and metabolic syndrome. Aim: To assess the prevalence and risk factors associated with OSAS in Chilean adults. Material and Methods: A standardized sleep questionnaire and respiratory polygraphy at home were conducted on adults aged 18 years or more, residing in the Metropolitan Region and enrolled in the 2016/17 National Health Survey. Results: Two-hundred and five people between 18 and 84 years old (46\% men, mean age 50 years) underwent overnight respiratory polygraphy at home. The estimated obstructive sleep apnea prevalence was 49\% (62\% men, $31 \%$ women) considering an apnea-hypopnea index $\geq 5$ respiratory events/hour, and $16 \%$ (21\% men, 13\% women) considering an apnea-hypopnea index $\geq 15$ respiratory events/hour. The prevalence of obstructive sleep apnea continuously increased along with age for men and women, with a later onset for women. Age, gender, body mass index, cervical and waist circumference, snoring, reporting of apnea by proxies, self-reported cardiovascular and metabolic diseases such as hypertension, diabetes and dyslipidemia, were significantly associated with OSAS. No association was found with insomnia and daytime sleepiness. Conclusions: The prevalence and risk factors associated to obstructive sleep apnea syndrome were high among these adults.

(Rev Med Chile 2020; 148: 895-905)

Key words: Epidemiology; Hypertension, Diabetes, Dyslipidemia; Prevalence; Risk Factors; Sleep Apnea, Obstructive; Sleep Apnea Syndromes.

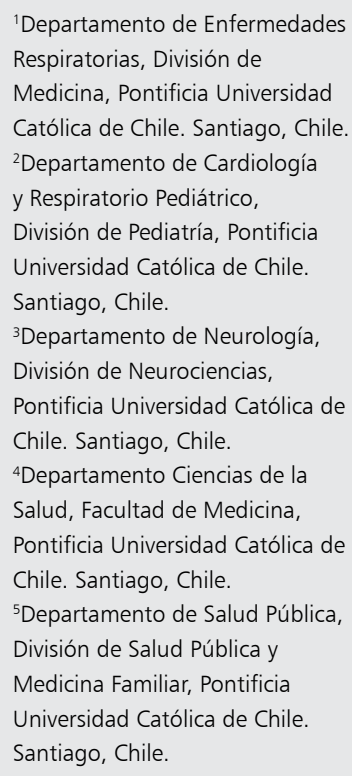

Trabajo no recibió financiamiento. Los autores declaran no tener conflictos de interés.

Recibido el 11 de marzo de 2019 aceptado el 1 de julio de 2020 .

Correspondencia a:

Dr. Fernando Saldías Peñafiel Departamento de Enfermedades Respiratorias, División de Medicina - Facultad de Medicina, Pontificia Universidad Católica de Chile. Santiago, Chile. fsaldias@med.puc.cl

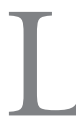

os trastornos respiratorios del sueño, especialmente el síndrome de apnea-hipopnea obstructiva del sueño (SAHOS), han adquirido gran relevancia e interés en la comunidad científica debido a sus graves consecuencias para la salud humana ${ }^{1}$. Este síndrome se caracteriza por episodios recurrentes de oclusión parcial o completa de la vía aérea superior durante el sueño, de origen multifactorial, que ocasiona fragmentación del sueño, episodios de hipoxemia e hipercarbia asociados a liberación de catecolaminas y hormonas de estrés, que ocasionan síntomas neuropsiquiátricos, deterioro en la calidad de vida y aumento en el riesgo cardiovascular de los enfermos ${ }^{2-4}$. Esta condición se manifiesta por ronquido intenso, pausas respiratorias durante 
el sueño, somnolencia diurna excesiva y sueño poco reparador ${ }^{1-4}$. Los pacientes con SAHOS moderado-grave tiene mayor riesgo que la población general de presentar hipertensión arterial refractaria a tratamiento, cardiopatía coronaria, arritmias, enfermedad cerebrovascular, insuficiencia cardíaca congestiva, resistencia a la insulina, síndrome metabólico, disminución de la libido o impotencia, deterioro cognitivo, disminución de la productividad laboral y mayor riesgo de accidentabilidad $^{5-11}$. El SAHOS suele coexistir con enfermedades cardiovasculares, respiratorias y metabólicas crónicas, aumentando el riesgo de base de estas enfermedades y dificultando su control clínico ${ }^{1-9}$.

Los estudios poblacionales sugieren que esta entidad afecta a 22\% (rango: 9\%-37\%) de los varones y $17 \%$ (rango: $4 \%-50 \%$ ) de las mujeres, especialmente entre la tercera y sexta década de la vida, asociado a factores de riesgo conocidos, tales como obstrucción de la vía aérea superior, obesidad, trastornos endocrinos (menopausia, hipotiroidismo, acromegalia, síndrome de ovario poliquístico) y alteraciones anatómicas del macizo maxilofacial (micrognatia, retrognatia) 1-4,12-16. En Chile, no se han realizado estudios poblacionales que permitan establecer la prevalencia de trastornos respiratorios del sueño (SAHOS) en la población adulta. En el estudio Platino, realizado en adultos mayores de 40 años de la Región Metropolitana, se estimó una prevalencia de SAHOS mediante cuestionario de sueño cercana a $6,4 \%$ de la población $(8,8 \%$ de los varones y $5 \%$ de las mujeres $)^{17}$. En la Encuesta Nacional de Salud 2009/10, se estimó mediante un cuestionario de sueño estandarizado, una prevalencia de SAHOS de 4,6\% en la población mayor de 15 años (5,3\% de los varones y $3,9 \%$ de las mujeres), con gradiente educacional inversa e incremento con la edad ${ }^{18}$.

El propósito del estudio es estimar la prevalencia de los trastornos respiratorios del sueño (SAHOS) en la población adulta chilena mediante un cuestionario de sueño estandarizado y la realización de poligrafías respiratorias en el hogar en una muestra poblacional seleccionada de la Región Metropolitana.

\section{Sujetos y Métodos}

Estudio transversal analítico que examinó los trastornos respiratorios del sueño (SAHOS) en una muestra (n: 205 sujetos) seleccionada entre los residentes de la Región Metropolitana que participaron en la Encuesta Nacional de Salud 2016-2017 (ENS 2016/17). En la ENS 2016/17 se seleccionó una muestra aleatoria compleja, estratificada y multietápica por conglomerados, de 9.901 viviendas; la muestra fue diseñada con estratos geográficos y demográficos homogéneos con sobrerrepresentación de regiones distintas a la metropolitana, zonas rurales y adultos mayores. Estas modificaciones fueron compensadas en el momento de los análisis, tomando en consideración factores de expansión que consideran las probabilidades de selección en cada etapa, corrección por no respuesta y postestratificación demográfica consecuente con las proyecciones censales de la población chilena a junio de 2016. Se seleccionó un adulto $\geq 15$ años por vivienda, excluyendo a embarazadas, personas con deterioro cognitivo que no pudieran responder a la encuesta de salud o que no aceptaran participar en el estudio. Se trabajó con una muestra aleatoria de viviendas que proviene del marco censal 2002, actualizado a 2008 para la muestra urbana. Usando una raíz del efecto de diseño de 1.797, la muestra permite estimaciones con $95 \%$ de confianza y error relativo inferior a $30 \%$ para prevalencias nacionales superiores a $2 \%$. La muestra ENS es representativa de la población nacional de 15 o más años, hombres y mujeres, zonas rurales y urbanas y de todas las regiones de Chile. La encuesta tuvo una tasa de respuesta en la población elegible de $67 \%$ y la tasa de rechazo fue de $9,8 \%$. Durante la primera visita a la vivienda, 6.233 sujetos mayores de 15 años respondieron a los cuestionarios que examinaron los principales problemas de salud de la población, incluyendo los trastornos respiratorios del sueño. El cuestionario de sueño constaba de diez preguntas que examinaron la duración del sueño, la presencia de ronquido habitual, pausas respiratorias durante el sueño, somnolencia diurna, sueño poco reparador, insomnio, uso de fármacos hipnóticos y la escala de somnolencia de Epworth ${ }^{19}$. En la segunda visita, 5.520 sujetos ( $89 \%$ de la muestra) fueron entrevistados y examinados por una enfermera capacitada, realizando mediciones antropométricas (peso, talla, índice de masa corporal, circunferencia de cintura) y de presión arterial en reposo, y se obtuvieron muestras de laboratorio en 5.451 participantes, midiendo la 
glicemia, Hb glicosilada, colesterol total, HDL y LDL colesterol, triglicéridos, $\mathrm{TSH}_{\text {y }} \mathrm{T}_{4}$ libre séricas, entre otros. Se estimó el riesgo de SAHOS de la población empleando los cuestionarios $\mathrm{STOP}^{20}$ y STOP-Bang ${ }^{21}$ que han sido empleados en estudios poblacionales y otros contextos clínicos ${ }^{22}$.

En una submuestra de la Región Metropolitana, estratificada por factores de riesgo de SAHOS (ronquido habitual y pausas respiratorias presenciadas por testigos), se evaluaron 205 sujetos mayores de 18 años, a quienes se les realizó un cuestionario de sueño, mediciones antropométricas y una poligrafía respiratoria (PR) en el hogar. El estudio fue revisado y aprobado por el Comité de Ética en Investigación de la Facultad de Medicina de la Pontificia Universidad Católica de Chile.

En los sujetos que aceptaron participar en el estudio y firmaron el consentimiento informado se consignaron los siguientes antecedentes clínicos: edad, sexo, ocupación, nivel educacional, comorbilidades, consumo de tabaco, alcohol y fármacos, peso, talla, índice de masa corporal, circunferencia cervical y de cintura, presión arterial en posición sentada, historia de sueño (duración, presencia de ronquido, pausas respiratorias, somnolencia diurna, insomnio, sueño poco reparador), los cuestionarios $\mathrm{STOP}^{20}$ y STOP-Bang ${ }^{21}$, y la escala de somnolencia de Epworth $^{19}$.

\section{Estudio trastornos respiratorios del sueño}

La evaluación de los trastornos respiratorios del sueño se realizó en el domicilio de los sujetos enrolados con un cuestionario de sueño y un equipo de poligrafía respiratoria que cumplía con las exigencias de la Academia Americana de Medicina del Sueño (AASM) para los estudios de nivel III $^{23-25}$. El equipo mide el flujo aéreo oronasal, esfuerzo respiratorio torácico y abdominal, pulsioximetría, frecuencia cardiaca, posición corporal y ronquido. Se empleó el software sleepware G3 with integrated somnolyzer scoring solution en la lectura de los exámenes. La entrega y retiro de los dispositivos fue realizado por un profesional de la salud, entrenado y capacitado para esta función, quien además corroboró in situ, la existencia y adecuación del registro para su posterior lectura. La duración mínima del estudio requerida para el análisis fue cuatro horas. El análisis de la PR fue realizado manualmente por un médico especialista en trastornos respiratorios del sueño que desconocía los antecedentes clínicos y los resultados de los cuestionarios de sueño; consignando la duración del estudio, posición corporal, número de apneas e hipopneas obstructivas, centrales y mixtas, caída de la saturación arterial de oxígeno bajo $90 \%$ (CT90\%) y número de episodios de desaturación mayor de 4\%. Se calculó el número de eventos respiratorios (IAH) dividiendo el número total de apneas e hipopneas por el tiempo total de registro en horas. La ejecución e interpretación de los exámenes siguió las recomendaciones de la Academia Americana de Medicina del Sueño ${ }^{26}$. El diagnóstico y la gravedad del SAHOS se clasificó siguiendo las recomendaciones internacionales: registro normal (IAH $<5$ eventos/hora), SAHOS leve (IAH: 5-14,9), moderado (IAH: 15-29,9) y grave (IAH $\geq 30$ eventos/h).

\section{Análisis estadístico}

Los resultados fueron expresados como valores promedio \pm desviación estándar para las variables numéricas de distribución normal y en porcentaje para las medidas en escala nominal. Las variables de distribución desconocida fueron consignadas como mediana y rangos intercuartílicos. Las variables cualitativas fueron comparadas mediante la prueba de chi cuadrado y el test exacto de Fisher, y las variables continuas según su distribución con la prueba t de Student o la prueba de Mann-Whitney. Para ello se utilizaron los programas estadísticos SPSS 25.0 (SPSS Inc, IBM Company, Chicago) y Stata/SE for large database (Stata Survey Data Reference Manual, STATA Corporation, Texas). La estimación de la prevalencia de SAHOS según edad y sexo fue estimada a partir de la información clínica y los informes de las poligrafías respiratorias realizadas en la muestra seleccionada de la población adulta de la Región Metropolitana. La estimación de la prevalencia de SAHOS fue realizada en base a una muestra expandida de la población adulta de la Región Metropolitana ajustada por la edad, género, historia de ronquido, pausas respiratorias durante el sueño y somnolencia diurna. Se evaluaron las variables clínicas, sociodemográficas y reglas predictivas asociadas al riesgo de SAHOS en la muestra de la Región Metropolitana. Las diferencias entre las variables fueron consideradas significativas con un valor de $\mathrm{p}<0,05$. 


\section{Resultados}

Se encuestaron 847 sujetos $\geq 18$ años en la Región Metropolitana, edad media: 48,5 \pm 18,3 años (rango: 18-85), 38,4\% varones, un tercio eran fumadores activos y un tercio eran obesos (IMC $\geq 30 \mathrm{~kg} / \mathrm{m}^{2}$ ), las principales enfermedades crónicas autoreportadas fueron hipertensión arterial $(32,8 \%)$, diabetes mellitus $(14,2 \%)$, dislipidemia $(29 \%)$ y depresión $(30,5 \%)$. En el cuestionario de sueño, 59,2\% reportaron ronquido habitual, $21,7 \%$ apneas presenciadas por testigos, $20,4 \%$ somnolencia diurna y $40,9 \%$ sueño poco reparador. En la Tabla 1 se describen las características sociodemográficas y clínicas de los 205 sujetos de la Región Metropolitana que se realizaron poligrafía respiratoria en el hogar $(24,2 \%$ de la muestra). En la Tabla 2 se describen las características clínicas, antropométricas, perfil metabólico y la historia de sueño reportada por la población adulta de la Región Metropolitana corregida por los factores de expansión.

La prevalencia de SAHOS moderado-grave en la población adulta de la Región Metropolitana fue $16,2 \%$ (IC 95\% 12,4\%-20,7\%), siendo más prevalente en los varones $(20,8 \%$, IC 95\% $14,8 \%$ $28,4 \%$ ) que en las mujeres (12,6\%, IC 95\% 8,4\%$18,6 \%)$. La prevalencia de trastornos respiratorios del sueño aumenta progresivamente con la edad en ambos sexos; sin embargo, en la mujer se eleva significativamente después de la menopausia (Tabla 3 y Figura 1). Las principales variables clínicas asociadas con el riesgo de SAHOS en la población fueron la edad, historia de ronquido habitual y pausas respiratorias durante el sueño, antecedente de hipertensión arterial y dislipidemia, el sobrepeso y la obesidad, y la circunferencia cervical y de cintura elevadas (Tabla 4). Los cuestionarios STOP y STOP-Bang se asociaron significativamente con el riesgo de SAHOS de la población. Mientras que el insomnio y la somnolencia diurna no se asociaron al riesgo de SAHOS.

La prevalencia de SAHOS fue más elevada en la población adulta residente en la Región Metropolitana con historia de ronquido habitual, antecedente de hipertensión arterial, diabetes mellitus y dislipidemia, obesidad central (IMC $\geq 30 \mathrm{~kg} / \mathrm{m}^{2}$ y circunferencia de cintura elevada) y somnolencia diurna excesiva objetivada por la escala de somnolencia de Epworth $\geq 10$ puntos (Tabla 5).

\section{Discusión}

Los principales hallazgos de este estudio fueron: 1) Alrededor de la mitad de la población adulta de la Región Metropolitana presenta trastornos respiratorios del sueño; siendo 16,2\% de magnitud moderada-severa; 2) Los trastornos respiratorios del sueño son más frecuentes en varones, especialmente entre la cuarta y sexta década de la vida, y en la mujer se eleva significativamente después de la menopausia; 3) Las principales variables clínicas asociadas al riesgo de SAHOS en la población adulta fueron la edad, sexo masculino, obesidad central, historia de ronquido y pausas respiratorias durante el sueño, antecedente de hipertensión arterial, diabetes mellitus y dislipidemia.

Este estudio nos permitió estimar la prevalencia de SAHOS en la población adulta de la Región Metropolitana, realizando cuestionario de sueño estandarizado, mediciones antropométricas y poligrafía respiratoria en el hogar a alrededor del $25 \%$ de la muestra examinada en la Encuesta $\mathrm{Na}$ cional de Salud 2016/17. La prevalencia estimada de SAHOS moderada-severa (IAH $\geq 15$ eventos respiratorios/hora) fue $16,2 \%$ en la población adulta (IC 95\% 12,4\%-20,7\%), los cuales deberían ser diagnosticados y tratados en forma oportuna y apropiada para lograr alivio sintomático, mejorar la calidad de vida y disminuir el riesgo cardiovascular o de accidentabilidad $d^{1,3,4,27}$. Similar a lo descrito en otros estudios ${ }^{14,16,28,29}$, la mayoría de los pacientes con SAHOS no habían sido diagnosticados; de hecho, sólo el $2 \%$ de los sujetos encuestados en la Región Metropolitana reportaron esta condición entre sus comorbilidades.

En los estudios poblacionales realizados en Europa, Asia y América se han reportado prevalencias variables de SAHOS ${ }^{16,29}: 22 \%$ (rango: 9\%-37\%) en varones y $17 \%$ (rango: $4-50 \%$ ) en mujeres, lo cual estaría determinado por las características genéticas de la población examinada, la presencia de factores de riesgo asociados a SAHOS (obesidad, hipertensión arterial, diabetes mellitus, síndrome metabólico), criterios diagnósticos (definición de apnea, hipopnea, desaturación), equipos utilizados en los estudios (polisomnografía en el laboratorio de sueño o poligrafía respiratoria en el hogar) y diseño metodológico o sistema de muestreo empleado en los estudios poblacionales. La prevalencia de SAHOS moderada-grave reportada en nuestro estudio fue similar a la comunicada 
Tabla 1. Características clínicas de la muestra de la Región Metropolitana de la Encuesta Nacional de Salud 2016/17 y de la submuestra de la Región Metropolitana a quienes se realizó poligrafía respiratoria en el hogar. Datos crudos, sin expansión muestral

\begin{tabular}{|c|c|c|c|c|}
\hline \multirow[b]{2}{*}{ Características } & \multicolumn{2}{|c|}{ Región Metropolitana } & \multicolumn{2}{|c|}{ Estudio del sueño en el hogar } \\
\hline & $n(X \pm D E)$ & $\begin{array}{c}\text { Porcentaje } \\
\text { (Rango) }\end{array}$ & n $(X \pm D E)$ & $\begin{array}{c}\text { Porcentaje } \\
\text { (Rango) }\end{array}$ \\
\hline $\mathrm{n}$ & 847 & & 205 & \\
\hline Edad (años) & $48,5 \pm 18,3$ & $18-85$ & $50,7 \pm 15,0$ & $18-84$ \\
\hline Género (Masculino-Femenino) & $325-522$ & $38,4-61,6$ & $95-110$ & $46,3-53,7$ \\
\hline Nivel educacional (A-B-C) & $157-409-280$ & $18,6-48,3-33,1$ & $28-108-69$ & $13,7-52,7-33,6$ \\
\hline $\begin{array}{l}\text { Consumo de tabaco } \\
\text { (F. activo-Exfumador-No fuma) }\end{array}$ & $290-270-287$ & $34,2-31,9-33,9$ & $67-82-56$ & $32,7-40,0-27,3$ \\
\hline Consumo de alcohol & $562 / 774$ & 72,6 & $154 / 205$ & 75,1 \\
\hline Menopausia & $198 / 522$ & 37,9 & $49 / 110$ & 44,5 \\
\hline Cardiopatía coronaria & $41 / 844$ & 4,9 & 15 & 7,3 \\
\hline Enfermedad cerebrovascular & $35 / 844$ & 4,1 & 12 & 5,8 \\
\hline Hipertensión arterial & $278 / 847$ & 32,8 & 77 & 37,6 \\
\hline Diabetes mellitus & $120 / 847$ & 14,2 & 35 & 17,1 \\
\hline Dislipidemia & $246 / 847$ & 29,0 & 88 & 42,9 \\
\hline Asma bronquial & $44 / 772$ & 5,7 & 12 & 5,9 \\
\hline Depresión & $257 / 844$ & 30,5 & 68 & 33,1 \\
\hline SAHOS & $19 / 774$ & 2,5 & ------ & ------ \\
\hline Peso $(\mathrm{Kg})$ & $74,2 \pm 15,0$ & $39,3-146,9$ & $77,7 \pm 16,2$ & $48,1-146,9$ \\
\hline Talla $(\mathrm{cm})$ & $161,0 \pm 9,6$ & $134,5-193$ & $162,3 \pm 9,7$ & $140-193$ \\
\hline Índice de masa corporal $\left(\mathrm{kg} / \mathrm{m}^{2}\right)$ & $28,6 \pm 5,1$ & $16,7-58,5$ & $29,4 \pm 5,3$ & $18,5-58,5$ \\
\hline $\mathrm{IMC} \geq 30 \mathrm{~kg} / \mathrm{m}^{2}$ & $266 / 772$ & $34,5 \%$ & $81 / 199$ & $40,7 \%$ \\
\hline Circunferencia cervical (cm) & ------- & ------- & $37,9 \pm 4,3$ & $29-55$ \\
\hline Circunferencia de cintura $(\mathrm{cm})$ & $93,9 \pm 13,4$ & $52,1-151,9$ & $96,2 \pm 13,4$ & $70,5-151,9$ \\
\hline Presión arterial sistólica (mmHg) & $124,5 \pm 21,3$ & $86-234$ & $124,9 \pm 18,3$ & $86-203$ \\
\hline Presión arterial diastólica $(\mathrm{mmHg})$ & $74,1 \pm 10,3$ & $49-110$ & $75,2 \pm 9,6$ & $52-98$ \\
\hline Glicemia en ayunas (mg/dL) & $97,4 \pm 34,7$ & $64-498$ & $97,9 \pm 37,4$ & $72-442$ \\
\hline Colesterol sérico (mg/dL) & $179,5 \pm 40,8$ & $93-410$ & $184,9 \pm 46,1$ & $98-410$ \\
\hline Creatininemia (mg/dL) & $0,79 \pm 0,38$ & $0,39-7,37$ & $0,77 \pm 0,17$ & $0,45-1,29$ \\
\hline Duración del sueño (horas) & $6,9 \pm 1,6$ & $3-12$ & $6,7 \pm 1,7$ & $3-12$ \\
\hline Roncador habitual & $486 / 821$ & 59,2 & 157 & 76,6 \\
\hline Apneas presenciadas & $169 / 778$ & 21,7 & 103 & 50,2 \\
\hline Somnolencia diurna excesiva & $173 / 847$ & 20,4 & 56 & 27,3 \\
\hline Sueño poco reparador & $346 / 847$ & 40,9 & 96 & 46,8 \\
\hline Riesgo de accidentes de tránsito & $7 / 492$ & 1,4 & $2 / 143$ & 1,4 \\
\hline Insomnio & $59 / 772$ & 7,6 & 21 & 10,2 \\
\hline Uso de hipnóticos & $113 / 846$ & 13,4 & 23 & 11,2 \\
\hline Escala de Epworth & $3,9 \pm 4,2$ & $0-21$ & $4,7 \pm 4,7$ & $0-21$ \\
\hline
\end{tabular}

Nota: $\mathrm{X} \pm \mathrm{DE}$ : Promedio \pm Desviación estándar; Nivel educacional: A: Menos de 8 años de estudio, B: 8-12 años, C: Más de 12 años de estudios; SAHOS: Síndrome de apnea-hipopnea obstructiva del sueño; IMC: Índice de masa corporal. 
Tabla 2. Prevalencia de factores de riesgo asociados al síndrome de apneas obstructivas del sueño ajustados a la población adulta de la Región Metropolitana. Encuesta Nacional de Salud 2016/17

\begin{tabular}{|c|c|}
\hline Características & $\begin{array}{l}\text { Región Metropolitan } \\
\text { Porcentaje (IC 95\%) }\end{array}$ \\
\hline $\mathrm{n}$ & 5.136 .745 \\
\hline Edad (años) & $18-85$ \\
\hline Consumo de tabaco (fumador activo) & $37,23(32,83-41,86)$ \\
\hline Consumo de alcohol & $75,96(71,41-79,99)$ \\
\hline Cardiopatía coronaria & $3,31(2,13-5,13)$ \\
\hline Enfermedad cerebrovascular & $3,34(2,09-5,31)$ \\
\hline Hipertensión arterial & $26,37(22,70-30,40)$ \\
\hline Diabetes mellitus & $11,54(9,03-14,63)$ \\
\hline Dislipidemia & $26,27(22,42-30,53)$ \\
\hline Depresión & $27,34(23,45-31,61)$ \\
\hline Roncador habitual & $61,20(55,58-66,54)$ \\
\hline Reporte de apneas & $22,37(18,43-26,87)$ \\
\hline Somnolencia diurna excesiva & $21,37(17,79-25,46)$ \\
\hline Sueño poco reparador & $44,25(39,32-49,29)$ \\
\hline Insomnio & $7,49(5,50-10,13)$ \\
\hline Uso de hipnóticos & $12,74(9,73-16,52)$ \\
\hline Accidentes de tránsito por somnolencia diurna & $1,24(0,41-3,72)$ \\
\hline Índice masa corporal $\geq 30 \mathrm{~kg} / \mathrm{m}^{2}$ & $33,11(28,17-38,47)$ \\
\hline $\begin{array}{l}\text { Circunferencia de cintura } \\
\text { Hombre } \geq 102 \mathrm{~cm} \text { - Mujer } \geq 88 \mathrm{~cm}\end{array}$ & $43,19(38,16-48,38)$ \\
\hline Escala de Epworth $>10$ & $6,30(4,07-9,63)$ \\
\hline Presión arterial sistólica $\geq 135$ mmHg & $18,01(14,81-21,73)$ \\
\hline Presión arterial diastólica $\geq 80 \mathrm{mmHg}$ & $24,93(21,31-28,9)$ \\
\hline Glicemia $\geq 100$ mmHg & $17,26(14,24-20,78)$ \\
\hline $\begin{array}{l}\text { HDL Colesterol } \\
\text { Hombre }<40 \text { mg/dL - Mujer }<50 \text { mg/dL }\end{array}$ & $47,15(41,67-52,71)$ \\
\hline Triglicéridos $\geq 150 \mathrm{mg} / \mathrm{dL}$ & $34,97(29,71-40,63)$ \\
\hline
\end{tabular}

Nota: Porcentajes e intervalos de confianza del 95\%. Estimación en base a una muestra expandida de la población adulta de la Región Metropolitana. Datos faltantes no son considerados al estimar las proporciones.

por Tufik y cols. en la ciudad de Sao Paulo, Brasil $(16,9 \%)^{30}$, Nakayama-Ashida y cols. en Osaka, Japón $(22,3 \% \text { en varones })^{31}$, Franklin y cols. en Uppsala, Suecia $(20 \% \text { en mujeres) })^{32}$, Johansson y cols. en el municipio rural de Kinda, Suecia $(23 \% \text { en ancianos })^{33}$ y Fietze y cols. en Pomerania al noreste de Alemania (21,2\% global, $29,7 \%$ en varones y $13,2 \%$ en mujeres $)^{34}$.
En nuestro estudio la prevalencia de SAHOS fue más elevada en varones (relación 2:1) y aumenta con la edad hasta la sexta década de la vida, similar a lo descrito en otros estudios poblacionales ${ }^{16,29}$. La mayor frecuencia de SAHOS en varones ha sido atribuida a efectos de las hormonas sexuales en la función de los músculos faríngeos y colapsabilidad de la vía aérea superior, diferencias 
Tabla 3. Prevalencia de síndrome de apnea-hipopnea obstructiva del sueño según sexo y edad ajustados a la población adulta de la Región Metropolitana

Prevalencia de SAHOS según sexo

\begin{tabular}{|cccc|}
\hline Sexo & $\begin{array}{c}\text { SAHOS } \\
\text { (IAH } \geq \mathbf{5} \text { ev/h) } \\
\text { Porcentaje (IC 95\%) }\end{array}$ & $\begin{array}{c}\text { SAHOS moderado-grave } \\
\text { (IAH } \geq \mathbf{1 5} \text { ev/h) } \\
\text { Porcentaje (IC 95\%) }\end{array}$ & $\begin{array}{c}\text { SAHOS grave } \\
\text { (IAH } \geq \mathbf{3 0 ~ e v / h )} \\
\text { Porcentaje (IC 95\%) }\end{array}$ \\
\hline Masculino & $62,27 \%(32,74-84,84 \%)$ & $20,75 \%(14,77-28,35 \%)$ & $8,71(4,93-14,94)$ \\
\hline Femenino & $31,05 \%(25,22-37,55 \%)$ & $12,61 \%(8,36-18,58 \%)$ & $3,10(1,30-7,21)$ \\
\hline Global & $49,40 \%(28,80-70,20 \%)$ & $16,15 \%(12,42-20,73 \%)$ & $5,61 \%(3,48-8,94)$ \\
\hline
\end{tabular}

Prevalencia de SAHOS según grupos etarios

\begin{tabular}{|cccc|}
\hline $\begin{array}{c}\text { Edad } \\
\text { (años) }\end{array}$ & $\begin{array}{c}\text { SAHOS } \\
\text { (IAH } \geq \mathbf{5} \text { ev/h) } \\
\text { Porcentaje (IC 95\%) }\end{array}$ & $\begin{array}{c}\text { SAHOS moderado-grave } \\
\text { (IAH } \geq \mathbf{1 5} \text { ev/h) } \\
\text { Porcentaje (IC 95\%) }\end{array}$ & $\begin{array}{c}\text { SAHOS grave } \\
\text { (IAH } \geq \mathbf{3 0 ~ e v / h )} \\
\text { Porcentaje (IC 95\%) }\end{array}$ \\
\hline $18-24$ & $15,77(2,41-58,71)$ & ------- & $-1,26(0,75-28,93)$ \\
\hline $25-34$ & $35,22(18,19-57,06)$ & $15,28(5,17-37,34)$ & $5,86(2,07-15,48)$ \\
\hline $35-44$ & $30,99(21,65-42,20)$ & $9,63(4,44-19,62)$ & $17,75(7,66-35,95)$ \\
\hline $45-54$ & $71,88(54,12-84,61)$ & $43,28(26,68-61,54)$ & $4,51(1,57-12,26)$ \\
\hline $55-64$ & $61,23(31,60-84,38)$ & $19,73(12,58-29,56)$ & $7,83(2,44-22,36)$ \\
\hline
\end{tabular}

Nota: Porcentajes e intervalos de confianza del 95\%. Estimación de la prevalencia de SAHOS en base a una muestra expandida de la población adulta de la Región Metropolitana ajustada por la historia de ronquido, pausas respiratorias durante el sueño y somnolencia diurna.

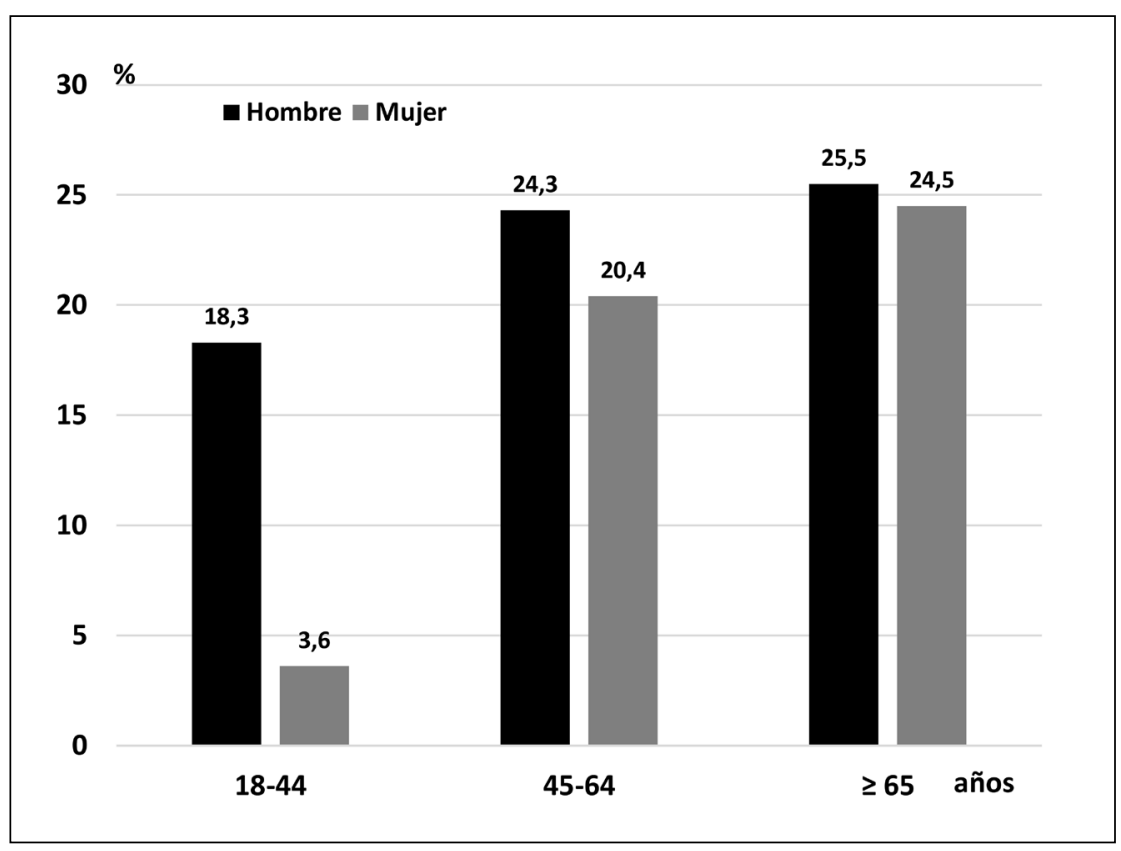

Figura 1. Prevalencia de síndrome de apnea-hipopnea obstructiva del sueño de magnitud moderada-severa según sexo y grupo etario ajustados a la población adulta de la Región Metropolitana. 
Tabla 4. Factores de riesgo asociados al síndrome de apnea-hipopnea obstructiva del sueño en la población adulta de la Región Metropolitana

\begin{tabular}{|c|c|c|}
\hline IAH $\geq 5$ eventos/hora & Odds ratio (IC 95\%) & $\mathbf{p}$ \\
\hline $\begin{array}{l}\text { Edad (años) } \\
\text { 18-44 años } \\
\text { 45-64 años } \\
\geq 65 \text { años }\end{array}$ & $\begin{array}{c}1,0 \text { (referencia) } \\
3,56(1,87-6,77) \\
5,56(2,29-13,45)\end{array}$ & $\begin{array}{l}0,0001 \\
0,0001\end{array}$ \\
\hline Sexo masculino vs femenino & $1,68(0,95-2,95)$ & 0,0722 \\
\hline Ronquido habitual & $2,53(1,31-4,88)$ & 0,0060 \\
\hline Reporte de apneas & $2,31(1,31-4,08)$ & 0,0040 \\
\hline Somnolencia diurna & $1,22(0,65-2,29)$ & 0,5353 \\
\hline Hipertensión arterial & $2,14(1,18-3,89)$ & 0,0129 \\
\hline Diabetes mellitus & $0,91(0,44-1,90)$ & 0,8037 \\
\hline Dislipidemia & $2,56(1,42-4,61)$ & 0,0017 \\
\hline Depresión & $0,93(0,53-1,62)$ & 0,7871 \\
\hline $\begin{array}{l}\text { Estado nutritivo } \\
\left.\text { Normal (IMC }<25 \mathrm{~kg} / \mathrm{m}^{2}\right) \\
\left.\text { Sobrepeso (IMC: } 25-29,9 \mathrm{~kg} / \mathrm{m}^{2}\right) \\
\left.\text { Obesidad (IMC } \geq 30 \mathrm{~kg} / \mathrm{m}^{2}\right)\end{array}$ & $\begin{array}{l}1,0 \text { (referencia) } \\
2,36(1,01-5,54) \\
8,66(3,47-21,6)\end{array}$ & $\begin{array}{l}0,0498 \\
0,0001\end{array}$ \\
\hline $\begin{array}{l}\text { Circunferencia de cintura } \\
\text { Hombre } \geq 102 \mathrm{~cm} \\
\text { Mujer } \geq 88 \mathrm{~cm}\end{array}$ & $3,28(1,80-5,96)$ & 0,0001 \\
\hline $\begin{array}{l}\text { Circunferencia cervical } \\
\text { Hombre }>39 \mathrm{~cm} \\
\text { Mujer }>35 \mathrm{~cm}\end{array}$ & $4,76(2,59-8,76)$ & 0,0001 \\
\hline Escala de Epworth $>10$ & $1,80(0,71-4,54)$ & 0,2154 \\
\hline Cuestionario STOP $\geq 2$ & $3,83(2,10-6,99)$ & 0,0001 \\
\hline Cuestionario STOP Bang $\geq 3$ & $3,72(1,98-6,98)$ & 0,0001 \\
\hline IAH $\geq 15$ eventos/hora & Odds ratio (IC 95\%) & $\mathbf{p}$ \\
\hline $\begin{array}{l}\text { Edad (años) } \\
\text { 18-44 años } \\
45-64 \text { años } \\
\geq 65 \text { años }\end{array}$ & $\begin{array}{l}1,0 \text { (referencia) } \\
3,20(1,45-7,06) \\
2,76(1,06-7,14)\end{array}$ & $\begin{array}{l}0,0040 \\
0,0371\end{array}$ \\
\hline Sexo masculino vs femenino & $1,49(0,80-2,78)$ & 0,2075 \\
\hline Ronquido habitual & $2,07(0,90-4,76)$ & 0,0866 \\
\hline Reporte de apneas & $1,64(0,87-3,07)$ & 0,1242 \\
\hline Somnolencia diurna & $1,32(0,67-2,60)$ & 0,4243 \\
\hline Hipertensión arterial & $1,82(0,97-3,42)$ & 0,0629 \\
\hline Diabetes mellitus & $1,86(0,86-4,01)$ & 0,1147 \\
\hline Dislipidemia & $1,33(0,71-2,48)$ & 0,3671 \\
\hline Depresión & $1,09(0,58-2,03)$ & 0,7929 \\
\hline $\begin{array}{l}\text { Estado nutritivo } \\
\left.\text { Normal (IMC }<25 \mathrm{~kg} / \mathrm{m}^{2}\right) \\
\left.\text { Sobrepeso (IMC: } 25-29,9 \mathrm{~kg} / \mathrm{m}^{2}\right) \\
\left.\text { Obesidad (IMC } \geq 30 \mathrm{~kg} / \mathrm{m}^{2}\right)\end{array}$ & $\begin{array}{l}1,0 \text { (referencia) } \\
1,10(0,36-3,35) \\
3,85(1,35-10,9)\end{array}$ & $\begin{array}{l}0,8611 \\
0,0118\end{array}$ \\
\hline $\begin{array}{l}\text { Circunferencia de cintura } \\
\text { Hombre } \geq 102 \mathrm{~cm} \\
\text { Mujer } \geq 88 \mathrm{~cm}\end{array}$ & $2,19(1,15-4,19)$ & 0,0174 \\
\hline $\begin{array}{l}\text { Circunferencia cervical } \\
\text { Hombre }>39 \mathrm{~cm} \\
\text { Mujer }>35 \mathrm{~cm}\end{array}$ & $2,81(1,38-5,70)$ & 0,0044 \\
\hline Escala de Epworth $>10$ & $2,22(0,92-5,36)$ & 0,0749 \\
\hline Cuestionario STOP $\geq 2$ & $2,08(1,03-4,20)$ & 0,0415 \\
\hline Cuestionario STOP Bang $\geq 3$ & $4,45(1,79-11,08)$ & 0,0013 \\
\hline
\end{tabular}




\begin{abstract}
Tabla 5. Estimación de la prevalencia de síndrome de apnea-hipopnea obstructiva del sueño de magnitud moderada-severa ajustados a la población adulta de la Región Metropolitana según factores de riesgo
\end{abstract}

\begin{tabular}{|c|c|c|}
\hline Categorías de riesgo & $\begin{array}{c}\text { Presente } \\
\text { Porcentaje (IC 95\%) }\end{array}$ & $\begin{array}{c}\text { Ausente } \\
\text { Porcentaje (IC 95\%) }\end{array}$ \\
\hline Ronquido habitual & $31,60(23,58-40,89)$ & $5,90(3,10-10,93)$ \\
\hline Reporte de apneas & $15,50(11,55-20,49)$ & $18,43(10,28-30,83)$ \\
\hline Somnolencia diurna & $17,65(11,23-26,64)$ & $15,70(11,34-21,33)$ \\
\hline Insomnio & $17,55(7,63-35,42)$ & $16,89(12,84-21,89)$ \\
\hline Tabaquismo & $25,71(17,94-35,38)$ & $18,25(12,24-26,33)$ \\
\hline Hipertensión arterial & $20,53(13,50-29,95)$ & $14,42(10,06-20,24)$ \\
\hline Diabetes mellitus & $24,33(14,98-36,97)$ & $14,28(10,43-19,24)$ \\
\hline Dislipidemia & $18,34(12,82-25,54)$ & $14,67(9,96-21,09)$ \\
\hline Depresión & $15,38(9,50-23,95)$ & $16,69(12,14-22,51)$ \\
\hline $\begin{array}{l}\text { Índice de masa corporal } \\
\text { Obesidad }\left(\geq 30 \mathrm{~kg} / \mathrm{m}^{2}\right) \\
\text { vs } \\
\text { Normal }\left(<25 \mathrm{~kg} / \mathrm{m}^{2}\right) \\
\text { Sobrepeso }\left(25-29,9 \mathrm{~kg} / \mathrm{m}^{2}\right)\end{array}$ & $25,75(17,68-35,90)$ & $\begin{array}{l}12,85(5,62-26,72) \\
10,45(6,08-17,37)\end{array}$ \\
\hline $\begin{array}{l}\text { Circunferencia de cintura } \\
\text { Hombre } \geq 102 \mathrm{~cm} \\
\text { Mujer } \geq 88 \mathrm{~cm}\end{array}$ & $\begin{array}{l}26,86(13,87-45,59) \\
31,85(20,08-46,50)\end{array}$ & $\begin{array}{c}17,97(11,10-27,76) \\
4,38(1,35-13,33)\end{array}$ \\
\hline Escala de Epworth $\geq 10$ & $30,07(16,19-48,92)$ & $15,83(11,73-21,02)$ \\
\hline
\end{tabular}

Nota: Porcentajes e intervalos de confianza del 95\%. Estimación de la prevalencia de SAHOS en base a una muestra expandida de la población adulta de la Región Metropolitana ajustada por la historia de ronquido, pausas respiratorias durante el sueño y somnolencia diurna.

en la distribución de la grasa corporal entre ambos sexos y cambios en la anatomía y estructura de la faringe que favorecerían su colapso durante el sueño $0^{35-39}$. La prevalencia de SAHOS aumenta con la edad independiente de otros factores de riesgo como la obesidad, hipertensión arterial y síndrome metabólico ${ }^{40,41}$. En la mujer aumenta significativamente la prevalencia de SAHOS después de la menopausia ${ }^{16,29}$. Las manifestaciones clínicas asociadas a SAHOS difieren en ambos sexos, lo cual retrasaría el proceso diagnóstico en las mujeres y limitaría su acceso al tratamiento, lo cual afectaría su calidad de vida y aumentaría su riesgo cardiovascular ${ }^{42,43}$. En las clínicas de sueño, la relación hombre/mujeres atendidos es cercana a 6-8:1, lo cual estaría determinado por diferencias en los factores de riesgo y manifestaciones clínicas asociadas a esta enfermedad.

Similar a lo descrito en otros estudios ${ }^{12-16,29-34}$, los principales factores de riesgo de SAHOS en la población fueron la edad, sexo masculino, historia de ronquido habitual, apneas presenciadas por testigos, obesidad central (IMC, circunferencia cervical y de cintura elevados) y la asociación con hipertensión arterial, diabetes mellitus y dislipidemia. Los cuestionarios de sueño STOP y STOP-Bang se asociaron significativamente al riesgo de SAHOS en la población adulta de la Región Metropolitana, lo cual había sido previamente reportado en estudios poblacionales y en sujetos de riesgo elevado derivados a las clínicas del sueño $0^{22,44,45}$.

Entre las principales limitaciones del estudio cabe mencionar el tamaño muestral de magnitud moderada que no permitió realizar el análisis de subpoblaciones de riesgo y el uso de equipos de poligrafía respiratoria que tienden a subestimar la magnitud del problema y no permiten precisar las características del fenómeno en las distintas fases del sueño. 
En resumen, los trastornos respiratorios del sueño son un problema altamente prevalente en la población adulta de la Región Metropolitana, está relacionado con la edad, sexo, obesidad de distribución central y algunas enfermedades cardiovasculares y metabólicas prevalentes en el mundo occidental, tales como la hipertensión arterial, diabetes mellitus y dislipidemia. El diagnóstico y tratamiento oportuno de esta condición mejora significativamente la calidad de vida y disminuye el riesgo cardiovascular de los enfermos con SAHOS moderado-grave.

\section{Referencias}

1. Mukherjee S, Patel SR, Kales SN, Ayas NT, Strohl KP, Gozal D, Malhotra A; American Thoracic Society ad hoc Committee on Healthy Sleep. An Official American Thoracic Society Statement: The Importance of Healthy Sleep. Recommendations and future priorities. Am J Respir Crit Care Med 2015; 191: 1450-8.

2. American Academy of Sleep Medicine Task Force. Sleep-related breathing disorders in adults: recommendations for syndrome definition and measurement techniques in clinical research. Sleep 1999; 22: 667-89.

3. Durán-Cantolla J, Puertas-Cuesta FJ, Pin-Arboledas G, Santa María-Cano J, el Grupo Español de Sueño (GES). Documento de consenso nacional sobre el síndrome de apneas-hipopneas del sueño. Arch Bronconeumol 2005; 41: 1-110.

4. Lloberes P, Durán-Cantolla J, Martínez-García MA, Marín JM, Ferrer A, Corral J, et al. Diagnosis and treatment of sleep apnea-hypopnea syndrome. Spanish Society of Pulmonology and Thoracic Surgery. Arch Bronconeumol 2011; 47: 143-56.

5. Parish JM, Somers VK. Obstructive sleep apnea and cardiovascular disease. Mayo Clin Proc 2004; 79: 1036-46.

6. Gottlieb DJ, Yenokyan G, Newman AB, O'Connor GT, Punjabi NM, Quan SF, et al. Prospective study of obstructive sleep apnea and incident coronary heart disease and heart failure: the sleep heart health study. Circulation 2010; 122: 352-60.

7. Cassar A, Morgenthaler TI, Rihal CS, Prasad A, Lennon RJ, Lerman LO, et al. Coronary endothelial function in patients with obstructive sleep apnea. Coron Artery Dis 2014; 25: 16-22.

8. Kim NH, Cho NH, Yun CH, Lee SK, Yoon DW, Cho HJ, et al. Association of obstructive sleep apnea and glucose metabolism in subjects with or without obesity. Diabetes Care 2013; 36: 3909-15.
9. Yaggi HK, Concato J, Kernan WN, Lichtman JH, Brass LM, Mohsenin V. Obstructive sleep apnea as a risk factor for stroke and death. N Engl J Med 2005; 353 : 2034-41.

10. Terán Santos J, Jiménez-Gómez A, Cordero-Guevara JA. The association between sleep apnea and the risk of traffic accidents. Cooperative Group Burgos-Santander. N Engl J Med 1999; 340: 847-51.

11. Young T, Finn L, Peppard PE, Szklo-Coxe M, Austin D, Nieto FJ, et al. Sleep disordered breathing and mortality: eighteen-year follow-up of the Wisconsin sleep cohort. Sleep 2008; 31: 1071-8.

12. Young T, Palta M, Dempsey J, Skatrud J, Weber S, Badr S. The occurrence of sleep-disordered breathing among middle-aged adults. N Engl J Med 1993; 328: 1230-35.

13. Young T, Shahar E, Nieto FJ, Redline S, Newman AB, Gottlieb DJ, et al. Predictors of sleep-disordered breathing in community-dwelling adults: the Sleep Heart Health Study. Arch Intern Med 2002; 162: 893-900.

14. Simpson L, Hillman DR, Cooper MN, Ward KL, Hunter M, Cullen S, et al. High prevalence of undiagnosed obstructive sleep apnoea in the general population and methods for screening for representative controls. Sleep Breath 2013; 17: 967-73.

15. Peppard PE, Young T, Barnet JH, Palta M, Hagen EW, Hla KM. Increased prevalence of sleep-disordered breathing in adults. Am J Epidemiol 2013; 177: 1006-14.

16. Franklin KA, Lindberg E. Obstructive sleep apnea is a common disorder in the population-a review on the epidemiology of sleep apnea. J Thoracic Dis 2015; 7: 1311-22.

17. Bouscoulet LT, Vázquez-García JC, Muiño A, Márquez M, López MV, de Oca MM, Talamo C, Valdivia G, Pertuze J, Menezes AM, Pérez-Padilla R; PLATINO Group. Prevalence of sleep related symptoms in four Latin American cities. J Clin Sleep Med 2008; 4: 579-85.

18. Encuesta Nacional de Salud ENS Chile 2009-2010. Ministerio de Salud, Chile. Páginas 496-500.

19. Johns MW. A new method for measuring daytime sleepiness: the Epworth sleepiness scale. Sleep 1991; 14: 540-5.

20. Chung F, Yegneswaran B, Liao P, Chung SA, Vairavanathan S, Islam S. STOP questionnaire: a tool to screen patients for obstructive sleep apnea. Anesthesiology 2008; 108: 812-21.

21. Chung F, Abdullah HR, Liao P. STOP-Bang Questionnaire: A practical approach to screen for obstructive sleep apnea. Chest 2016; 149: 631-8.

22. Nagappa M, Liao P, Wong J, Auckley D, Ramachandran SK, Memtsoudis S, et al. Validation of the STOP-Bang 
questionnaire as a screening tool for obstructive sleep apnea among different populations: A systematic review and meta-analysis. PLoS One 2015; 10: e0143697.

23. Collop NA, Anderson WM, Boehlecke B, Claman D, Goldberg R, Gottlieb DJ, et al. Clinical guidelines for the use of unattended portable monitors in the diagnosis of obstructive sleep apnea in adult patients. Portable Monitoring Task Force of the American Academy of Sleep Medicine. J Clin Sleep Med 2007; 3: 737-47.

24. El Shayeb M, Topfer LA, Stafinski T, Pawluk L, Menon D. Diagnostic accuracy of level 3 portable sleep tests versus level 1 polysomnography for sleep-disordered breathing: a systematic review and meta-analysis. CMAJ 2014; 186: E25-51.

25. Lux L, Boehlecke B, Lohr KN. Effectiveness of Portable Monitoring Devices for Diagnosing Obstructive Sleep Apnea: Update of a Systematic Review. Rockville (MD): Agency for Healthcare Research and Quality (US); 2004. AHRQ Technology Assessments.

26. Kapur VK, Auckley DH, Chowdhuri S, Kuhlmann DC, Mehra R, Ramar K, Harrod CG. Clinical Practice Guideline for Diagnostic Testing for Adult Obstructive Sleep Apnea: An American Academy of Sleep Medicine Clinical Practice Guideline. J Clin Sleep Med 2017; 13: 479-504.

27. Giles TL, Lasserson TJ, Smith B, White J, Wright JJ, Cates CJ. Continuous positive airways pressure for obstructive sleep apnoea in adults. Cochrane Database Syst Rev 2006; (1): CD001106.

28. Spence CD, Han CT, Morriso

n CT, Couture CD. High rate of undiagnosed obstructive sleep apnea in patients undergoing total joint arthroplasty. AANA J 2018; 86: 282-8.

29. Senaratna CV, Perret JL, Lodge CJ, Lowe AJ, Campbell $\mathrm{BE}$, Matheson MC, et al. Prevalence of obstructive sleep apnea in the general population: A systematic review. Sleep Med Rev 2017; 34: 70-81.

30. Tufik S, Santos-Silva R, Taddei JA, Bittencourt LR. Obstructive sleep apnea syndrome in the Sao Paulo Epidemiologic Sleep Study. Sleep Med 2010; 11: 441-6.

31. Nakayama-Ashida Y, Takegami M, Chin K, Sumi K, Nakamura T, Takahashi K, et al. Sleep-disordered breathing in the usual lifestyle setting as detected with home monitoring in a population of working men in Japan. Sleep 2008; 31: 419-25.

32. Franklin KA, Sahlin C, Stenlund H, Lindberg E. Sleep apnoea is a common occurrence in females. Eur Respir J 2013; 41: 610-5.
33. Johansson P, Alehagen U, Svanborg E, Dahlström U, Broström A. Sleep disordered breathing in an elderly community-living population: Relationship to cardiac function, insomnia symptoms and daytime sleepiness. Sleep Med 2009; 10: 1005-11.

34. Fietze I, Laharnar N, Obst A, Ewert R, Félix SB, García $\mathrm{C}$, et al. Prevalence and association analysis of obstructive sleep apnea with gender and age differences - Results of SHIP-Trend. J Sleep Res 2018: e12770.

35. Mohsenin V. Effects of gender on upper airway collapsibility and severity of obstructive sleep apnea. Sleep Med 2003; 4: 523-9.

36. Pillar G, Malhotra A, Fogel R, Beauregard J, Schnall R, White DP. Airway mechanics and ventilation in response to resistive loading during sleep: influence of gender. Am J Respir Crit Care Med 2000; 162: 1627-32.

37. Mohsenin V. Gender differences in the expression of sleep-disordered breathing: role of upper airway dimensions. Chest 2001; 120: 1442-7.

38. Whittle AT, Marshall I, Mortimore IL, Wraith PK, Sellar RJ, Douglas NJ. Neck soft tissue and fat distribution: comparison between normal men and women by magnetic resonance imaging. Thorax 1999; 54: 323-8.

39. Popovic RM, White DP. Upper airway muscle activity in normal women: influence of hormonal status. J Appl Physiol 1998; 84: 1055-62.

40. Bixler EO, Vgontzas AN, Ten Have T, Tyson K, Kales A. Effects of age on sleep apnea in men: I. Prevalence and severity. Am J Respir Crit Care Med 1998; 157: 144-8.

41. Bixler EO, Vgontzas AN, Lin HM, Ten Have T, Rein J, Vela-Bueno A, Kales A. Prevalence of sleep-disordered breathing in women: effects of gender. Am J Respir Crit Care Med 2001; 163 (3 Pt 1): 608-13.

42. Lin CM, Davidson TM, Ancoli-Israel S. Gender differences in obstructive sleep apnea and treatment implications. Sleep Med Rev 2008; 12: 481-96.

43. Ye L, Pien GW, Weaver TE. Gender differences in the clinical manifestation of obstructive sleep apnea. Sleep Med 2009; 10: 1075-84.

44. Saldías F, Gassmann J, Canelo A, Uribe J, Díaz O. Evaluación de los cuestionarios de sueño en la pesquisa de pacientes con síndrome de apneas obstructivas del sueño. Rev Med Chile 2018; 146: 1123-34.

45. Saldías F, Salinas G, Cortés J, Farías D, Peñaloza A, Aguirre C, Díaz O. Rendimiento de los cuestionarios de sueño en la pesquisa de pacientes adultos con síndrome de apnea obstructiva del sueño según sexo. Rev Med Chile 2019; 147: 1291-302. 